\title{
Investigation of rigid dynamic systems on the example of modelling a tape drive mechanism
}

\author{
Ruslan Nigay $^{1 *}$, Evgeny Nigay $^{1}$, and Lubov Mironova ${ }^{2}$ \\ ${ }^{1}$ Russian University of Transport (MIIT), Russia \\ ${ }^{2}$ Moscow Aviation Institute (State National Research University) Russia
}

\begin{abstract}
The features of modeling the dynamics of mechanical systems on the example of the operation of the tape drive mechanism related to real technological processes are stated. An approach to solving stiff systems of differential equations by the numerical-analytical method is noted. The approach is based on solving systems of higher-order differential equations using elementary functions using procedures for precision search for the roots of the characteristic polynomial of the system. A mathematical model of the tape drive mechanism of a VCR is given as an example of a precision electromechanical object.
\end{abstract}

\section{Introduction}

Currently, high-precision intelligent equipment is used in many technological processes. For example, in prototyping technologies, one of the important tasks is the delivery of material to the zone of layer-by-layer formation of an object. This material can be thread or special tape, which can be fed to the working chamber using a tape drive. The accuracy of the material feeding largely depends on the positioning accuracy of the actuator, and on the dynamic phenomena caused by the vibrations of the structural elements of the mechanism. Hence, it becomes necessary to study dynamic objects with increased requirements for a precision system of motion parameters and maximum consideration of all components of the system's motion. Such systems constitute rigid dynamical systems. The study of the features of dynamic phenomena in the processes of motion transmission is inextricably linked with the development of a mathematical model and its behavioural algorithm.

\subsection{Statement of the problem}

A special place among various dynamic objects is occupied by objects with increased requirements for the precision of the motion parameters, when it becomes necessary to take into account all the components of the system motion [1]. But,despite the fact that a sharp boundary between precision and non-precision systems cannot be drawn, there are a number of criteria by which it is customary to classify a system as precision. These signs include:

- high precision requirements for measurement metrology,

* Corresponding author: ruslan1010@mail.ru 
- high precision requirements for measurement metrology,high order of systems of differential equations describing their dynamics due to the need to take into account a larger number of components of the system motion,

- the need to use computer technology in the process of research and design, as well as management,

- manifestation of the property of rigidity in mechanisms.

The study of the features of dynamic phenomena in the processes of motion transmission is inextricably linked with the development of a mathematical model and its behavioural algorithm. Many studies of dynamic systems, adapted to specific technological processes, include various approaches to the construction of a mathematical model. For example, in [2-5], modeling of complex technological objects was carried out on the basis of the method using the well-known equations of the momentum in differential form for longitudinal and torsional vibrations. This approach made it possible to relate the fluctuations in the velocities and stresses in rather complex systems with both distributed parameters and significant nonlinearities.

It should be noted that a feature of the formalization of a complex technical process is the construction of a mathematical model for rigid dynamical systems using high-order differential equations. The manifested property of rigidity is that, in the observation interval, to describe the motion of the system, one has to use functions of two types: rapidly changing functions with large derivatives (fast motions) and slowly decaying functions with small derivatives (slow motions). Particular difficulties arise if the moments of the beginning of fast movements are not known in advance [6].

We present the results of modeling rigid dynamic systems using the example of a tape drive mechanism. The following approach is proposed. In fig. 1 shows a block diagram of the tape drive mechanism used in the tape (thread) reinforcement technology.

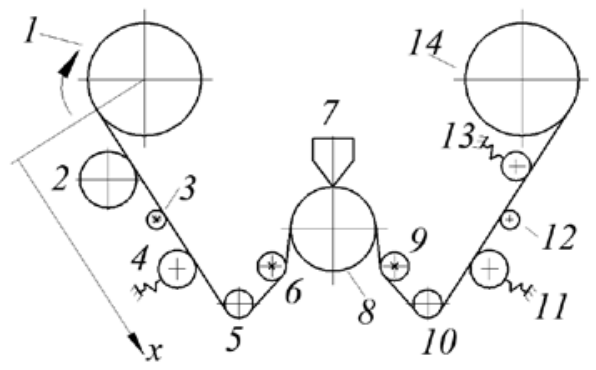

Fig. 1. Diagram of the tape drive mechanism: 1 is reeling device; 2 is drive shaft; 3, 6, 9, 12 are fixed guides; 4, 11, 13 are spring-loaded guides; 5, 10, 12 are movable guides; 7 is reinforcement device; 8 is drum; 14 is unwinding device.

The mechanism is a closed system. The peculiarity of the mechanism is in the precise supply of the tape to the reinforcement chamber for applying the reinforcement pattern by device 7.

When constructing a mathematical model of the movement of the tape drive, we make the following assumptions:

- some parts of the system are devoid of mass. their mass is very small compared to other parts of the system,

- systems with low weight are described as deformable inertial bonds (tape or thread),

- components that have comparable mass and properties of inertia are considered as material points, i.e. concentrated masses.

The generalized coordinates of the system will be small fluctuations in the speed of movement of the points of the tape relative to the stationary movement at the points of 
contact with the elements of the mechanism. Generalized coordinates are denoted by xi, where the $\mathrm{i}$-th index corresponding to the number of the element of the path to which the coordinate belongs.

The problem of determining the fluctuations in the speed of the belt is solved by compiling and solving a system of differential equations describing the motion of the conditional points of the belt itself.

\section{Derivation of Equations}

To construct a system of differential equations, we will use a procedure based on the Langrage equations of the second kind.

$$
\frac{d}{d t}\left(\frac{\partial T}{\dot{q}_{i}}\right)-\frac{\partial T}{\partial q_{i}}=Q_{i}, i=1,2, \ldots, s,
$$

where $T$ is the kinetic energy; $q_{i} \dot{q}$ is the generalized coordinate and its derivative; $Q_{i}$ is the generalized force; $s$ is the number of component variables in the system.

where $\mathrm{T}$ is the kinetic energy; qi is the generalized coordinate and its derivative; Qi is the generalized force; $\mathrm{s}$ is the number of component variables in the system.

We neglect the mass of the magnetic tape because of its smallness in comparison with the masses of the constituent elements of the mechanism. The interaction of the surfaces of the rotating elements with the belt can also be neglected, with the exception of dry friction forces acting in the direction of the belt movement.

In what follows, we will consider the movement of the tape in the longitudinal direction, assuming that the guiding elements of the mechanism do not allow lateral vibration of the tape. Rotational motion parameters, such as moments of inertia, torsional severity, damping coefficient of rotational motion, according to well-known rules, are brought to the translational motion of the belt.

When determining the potential energy of small vibrations of the system, we assume that the action of dry friction forces between the tape and the surface of the rotating elements of the mechanism exclude their movement relative to each other, i.e. there is no magnetic tape slippage.

The stationary motion of the tape is taken as the origin of the generalized coordinates. For the positive direction of reference of the generalized coordinates, we take the direction of stationary motion of the tape. For the positive direction of reading the generalized coordinates of the drum, guides and spring-loaded guides, we take the direction that coincides with the direction of movement of the tape.

With the stationary movement of the tape along the structural elements of the mechanism, all generalized coordinates are equal to zero. The adopted coordinate system makes it possible to directly monitor the behavior of the main indicator of the quality of the mechanism functioning - longitudinal oscillations of the belt speed.

In accordance with the kinematic diagram of the mechanism (Fig. 1), the potential energy of small displacements near the stationary motion are: elastic deformation of the belt, springs, drum and guides. We believe that it is infinite the small work of elastic deformation is the total differential of some force function $U$ [7] and $U$ is a function of the generalized coordinates

$$
U=U\left(q_{1}, q_{2}, \ldots, q_{n}\right)
$$

The potential energy of elastic deformation is expressed by the formula 


$$
W=\sum F_{i} d q_{i}=\sum \frac{\partial U}{\partial q_{i}} d q, F_{i}=\frac{\partial U}{\partial q_{1}} .
$$

Due to the small elastic deformation and translational motion of the tape, we write down the final formula

$$
W=\frac{1}{2} \sum F_{i} \Delta x=\frac{1}{2} \sum k_{i} x_{i} \Delta x .
$$

Here $F_{i}$ is the normal force, $F_{i}=k_{i} x_{i} ; \Delta x$ is displacement from equilibrium; $k_{i}$ is the stiffness of the structural element; $x_{i}$ is the current coordinate.

When the tape moves along the path of the mechanism, the change in the generalized coordinates is determined as the geometric distance between the points of contact of the tape with two adjacent elements. Therefore, the stiffness of the tape is also a function of the generalized coordinates and is expressed by the following formula

$$
k_{(t) i, i+1}=\frac{E s}{l_{i, i+1}+x_{i}+x_{i+1}},
$$

where $l_{i, i+1}$ is the length of the tape between the structural elements.

It should be noted that for springs the stiffness will be determined by a different formula

$$
k_{(s) i}=\frac{G d^{4}}{8 D^{3} n},
$$

where $G$ is the shear modulus; $d$ is the wire diameter; $D$ is diameter between the axes of the spring wire; $n$ is the number of turns.

The total kinetic energy of a mechanical system at a constant speed of movement is determined by the sum of the kinetic energies of its rotating nodes, i.e. reduced masses $m_{i}$ and is determined by the formula

$$
T=\frac{1}{2} \sum m_{i} x_{i}^{2} .
$$

Consider the resistance forces acting in the tape drive during its operation. According to the main feature, they are divided into dry and viscous friction forces. Dry friction forces appear at the points of contact of the belt with fixed elements (guides). These forces are complex and are described by nonlinear functions of time

$$
Q_{i}=v_{0} F_{i} \frac{d x_{i}}{d t} .
$$

Here $v_{0}$ is the stationary magnetic tape speed.

Substituting (8) into expression (1) and taking into account (7), we obtain a system of differential equations describing the non-stationary component of the belt motion.

Taking into account the fact that the system of Lagrange equations of the second kind was obtained when considering the quantities $x_{i}$ and $t_{i}$ of the first order of smallness, then the differential equations of motion are compiled accurate to quantities of the first order of smallness. Accordingly, their derivatives $\partial / \partial t\left(\partial T / x_{t}\right), \partial T / \partial x, \partial U / \partial x$ will also be quantities of the first order of smallness.

As an example, we write down the differential equation for the coiler 1. 


$$
m_{1} \frac{d^{2} x_{1}}{d t^{2}}-x_{1} k_{(t) 1,3}\left(x_{1}, x_{3}\right)=-\left[x_{3}+f_{1}\left(x_{2}\right) k_{(s) 1,3}-f_{1}\left(v_{0}-\frac{d x_{1}}{d t}\right)\right] .
$$

In a similar way, an equation can be drawn up for each structural element, having obtained a complete system of differential equations that describe the dynamics of the movement of the tape in the mechanism. This system is not linear. However, using wellknown linearization methods, for example, the expansion of the dry friction function in a Taylor series, we obtain a system of algebraic equations and in vector-matrix form it has the form

$$
\begin{aligned}
& \frac{d x}{d t}=A X+B U \\
& Y=C X+D U
\end{aligned}
$$

Here $X$ is a $19 \times 1$ state vector; $U$ is a $1 \times 1$ vector of control actions; $Y$ is a vector of output variables with a dimension of $14 \times 1 ; A, B, C, D$ are matrices of functional coefficients.

Thus, the modeling of the tape drive mechanism is reduced to the method of modeling rigid systems based on elementary functions with a procedure for high-precision search for the roots of the characteristic polynomial of the system [6].

The interactive technique for finding roots uses a numerical-analytical procedure that uses a combination of various iterative methods for finding roots: the methods of Newton, Danilevsky, Hitchcock, etc., combined with an estimate of the area of the roots and excluding the found root from further search. The program for finding the roots of the characteristic polynomial of a system of higher-order differential equations is described in the literature [8].

\section{Conclusions}

The considered method for modelling a mechanical system using the example of a tape drive mechanism and the proposed approach in the numerical-analytical method for modelling rigid dynamic systems gives an exact solution (with the accuracy of the computer discharge grid) with any step and for any moment in time. The formulas obtained allow using a computer to calculate a fairly wide range of dynamic problems adapted to technological processes in engineering. A correct assessment of the dynamics will make it possible to find rational modes of equipment operation using robotics and flexible automated production modules.

\section{References}

1. V. Afanas'ev, V. Kolmanovskij, V. Nosov, Mathematical theory of design of control systems (Nauka, Moscow, 1989)

2. L. Kondratenko, L. Mironova, V. Terekhov, On the question of the relationship between longitudinal and torsional vibrations in the manufacture of holes in the details, Vibroengineering Procedia, v. 12, pp. 6-11 (2017)

3. L. Kondratenko, L. Mironova, V. Dmitriev, Loss of stability of open two-link mechanisms. Vibroengineering Procedia, v. 25, pp. 220-225 (2019)

4. L. Mironova, L. Kondratenko, Method for the study of dynamic characteristics in the mechanisms of motion transmission, Vibroengineering Procedia, v. 25, pp. 214-219 (2019) 
5. L. Mironova, L. Kondratenko, Mathematical modeling of the processing of holes on CNC machines, Materials Today: Proceedings, v. 19(5), pp. $2354-2357$ (2019)

6. N. Malahov, R. Nigay, Numerical-analytical method for modeling rigid dynamic systems, J. Proceedings of higher educational institutions. Instrumentation. v. 34(8) pp. 5-13 (1991)

7. C. Lanczos, The Variational Principles of Mechanics. University of Toronto Press, Toronto (1949)

8. R. Nigay, E. Nigay, L. Mironova, Interactive search for the roots of the characteristic polynomial of a system of higher order differential equations, Certificate of state registration of a computer program 2020614389 (2020) 